\title{
Outcomes after Scarf Osteotomy for Treatment of Adult Hallux Valgus Deformity
}

\author{
Stephanie P. Adam DO, Steven C. Choung MD, \\ Yang Gu BS, Martin J. O’Malley MD
}

Received: 31 January 2010/ Accepted: 26 July 2010/Published online: 13 August 2010

(C) The Association of Bone and Joint Surgeons (B) 2010

\begin{abstract}
Background Many procedures have been developed to correct hallux valgus deformity using distal soft tissue realignment, metatarsal osteotomy, and periodically, a proximal phalanx osteotomy (Akin). The ideal metatarsal osteotomy allows for varying degrees of correction with reliable improvement in deformity and patient satisfaction. Questions/purposes We evaluated the results after scarf osteotomy with respect to American Orthopaedic Foot and Ankle Society (AOFAS) scores, patient satisfaction, radiographic results, and complications.

Patients and Methods We evaluated 29 patients (34 feet) during an 18-month period who underwent a unilateral scarf osteotomy combined with distal soft tissue realignment. Preoperative and postoperative AOFAS scores, patient satisfaction, and radiographic data were used to evaluate the effectiveness of the procedure. Complications were recorded. Minimal followup was 12 months (average, 26.4 months; range, 12-48 months).
\end{abstract}

Each author certifies that he or she has no commercial associations (eg, consultancies, stock ownership, equity interest, patent/licensing arrangements, etc) that might pose a conflict of interest in connection with the submitted article.

Each author certifies that his or her institution approved the human protocol for this investigation, that all investigations were conducted in conformity with ethical principles of research, and that informed consent for participation in the study was obtained.

This work was performed at Hospital for Special Surgery.

S. P. Adam ( $₫)$, Y. Gu, M. J. O’Malley

Department of Foot and Ankle Surgery, Hospital for Special

Surgery, 535 East 70th Street, New York, NY 10021, USA

e-mail: StephaniePAdam@gmail.com

S. C. Choung

Department of Orthopaedic Surgery, Jewett Orthopaedic Clinic,

Orlando, FL, USA
Results The mean AOFAS scores improved from 61.5 to 90.3. At final followup, $94 \%$ of patients were satisfied with the surgery. The hallux valgus angle improved from $34.6^{\circ}$ to $14.9^{\circ}$ and the intermetatarsal angle improved from $15.8^{\circ}$ to $7.2^{\circ}$ postoperatively. A combined Akin osteotomy was performed in only four cases. Complications included superficial wound infection (one), recurrence (two), and troughing (three).

Conclusions Our results suggest the scarf osteotomy produces improved AOFAS scores, high percentage of patient satisfaction, and effective correction of hallux valgus deformities. Using our scarf technique of rotation combined with translation minimizes the need for an Akin osteotomy while still obtaining good correction and avoids associated complications described in the literature.

Level of Evidence Level IV, therapeutic study. See Guidelines for Authors for a complete description of levels of evidence.

\section{Introduction}

Hallux valgus deformity consists of lateral subluxation of the first metatarsophalangeal joint, metatarsus primus varus, and lateral deviation of the sesamoids [18]. Surgical treatment of this deformity involves correction of alignment with an appropriately chosen metatarsal osteotomy and soft tissue releases [10]. Distal osteotomies of the first metatarsal primarily are advocated for mild to moderate hallux valgus deformities. For more severe deformities, more proximal first metatarsal osteotomies have been performed [22]. The role of the combined metaphysealdiaphyseal osteotomy has not been fully evaluated.

The scarf osteotomy is a Z-step cut in the first metatarsal bone used to reduce the increased intermetatarsal angle 
(IMA) of hallux valgus deformity. The term "scarf" is a carpentry term referring to a joint created by notching two pieces of wood. This osteotomy is used widely in France and the United States and continues to evolve and increase in popularity in the surgical community [19, 22]. Use of the proximal phalanx (Akin) osteotomy as an adjunct to the scarf osteotomy has been described frequently [7, 13, 15, 16]. It routinely is added after completing the scarf osteotomy and soft tissue reconstruction when the hallux is still in greater than $10^{\circ}$ valgus [14].

The indications for the scarf osteotomy are wide-ranging, from mild to severe deformities [2, 6, 7, 16]. Specific contraindications are severe first tarsometatarsal instability, osteoarthritis of the first metatarsophalangeal joint, and severe osteoporosis [6]. The advantages of this osteotomy over other shaft osteotomies are its inherent stability and rigid compression at the osteotomy site, which allows for immediate weightbearing and the option for bilaterality $[13,14,23]$. This technique also offers tremendous versatility, permitting plantar flexion to increase the load of the first ray, angular correction improving the IMA, and maintenance of the metatarsal length. The scarf osteotomy compares favorably with other bunion surgeries reported in the literature $[8,9]$, but results and complication rates vary tremendously from study to study $[1,4,7,13]$. The technically demanding nature of the procedure requires a steep learning curve. A keen awareness of the associated complications and meticulous attention to detail when performing this osteotomy are paramount to preventing problems.

In our study, we sought answers to the following questions: (1) Does the scarf osteotomy result in improved AOFAS scores and patient satisfaction compared with their preoperative condition? (2) Will the scarf osteotomy alone correct radiographic parameters, ie, hallux valgus angle (HVA) and IMA? (3) What are the associated complications of this procedure? (4) What are some technical pearls in surgical technique to obtain good correction and avoid complications?

\section{Patients and Methods}

This study was approved by our institution's review board, and informed consent was obtained from all patients. We evaluated and followed a total of 46 consecutive patients (51 feet), between May 2000 and December 2001, who underwent a unilateral scarf osteotomy. All osteotomies were performed by one surgeon (MJO). The main operative indication for the surgery was pain and deformity in the forefoot, especially near the first metatarsophalangeal joint, refractory to nonoperative management. Nonoperative management included accommodating shoes or orthotic devices. Our indications for a scarf osteotomy included hallux valgus deformity with a HVA of as much as $55^{\circ}$ and an IMA of $10^{\circ}$ to $24^{\circ}$. Patients excluded from having this procedure were those with severe first tarsometatarsal instability, osteoarthritis of the first metatarsophalangeal joint, severe osteoporosis, and open epiphyseal plates. Of the 46 consecutive patients ( 51 feet), we had complete data for 29 patients (34 feet), excluding those without preoperative AOFAS scores and/or radiographs. There were 27 women and two men with an average age of 55 years (range, 18-82 years). Minimum followup was 12 months (average, 26.4 months; range, 12-48 months).

Our technique included five steps: lateral release of the metatarsal-sesamoidal-phalangeal complex, resection of the medial eminence, scarf osteotomy, medial capsulorraphy, and combined procedures (ie, Akin). The distal soft tissue realignment (lateral release plus medial resection) was described previously [2].

The scarf osteotomy incorporated three cuts. Two preliminary Kirschner wires were placed to mark out the osteotomy site. One Kirschner wire was directed laterally in the metatarsal, $5 \mathrm{~mm}$ from the distal cartilage, 2 to $3 \mathrm{~mm}$ from the dorsal surface, and parallel to the articular surface. Another Kirschner wire was directed laterally in the proximal metaphysis parallel to the articular surface of the metatarsal base. The longitudinal cut was marked with a surgical pen connecting the two wires, and the length was determined by the degree of correction necessary (Fig. 1). With a longer longitudinal cut, a greater correction of the IMA was made possible. The dorsal cut was marked from the distal wire angled $45^{\circ}$ proximally exiting the dorsal cortex. The plantar cut was marked from the proximal wire angled $45^{\circ}$ distally exiting the plantar cortex. Hohmann retractors were placed dorsal and plantar on the metatarsal

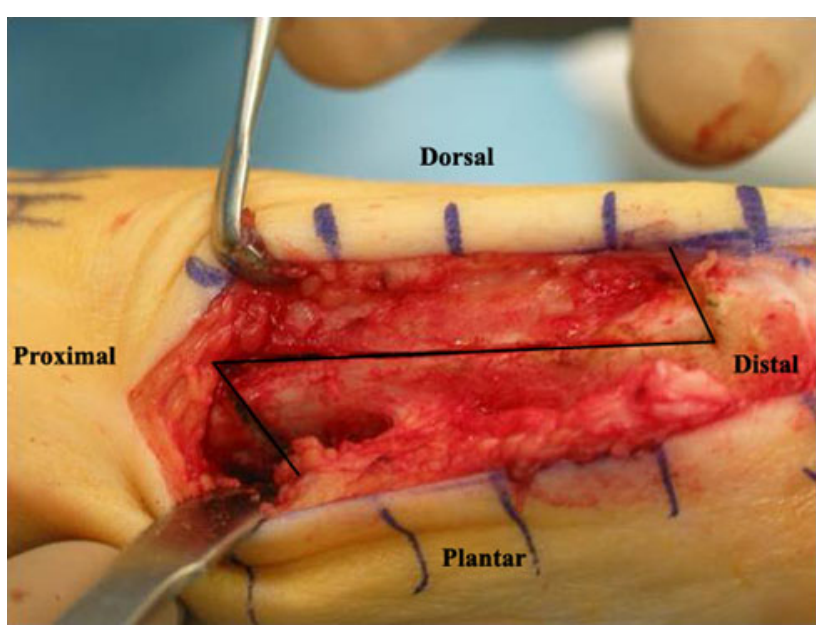

Fig. 1 A photograph shows the outline on the first metatarsal highlighting the scarf osteotomy cut. 
shaft to avoid injury to the extensor hallucis longus and flexor hallucis longus, respectively. The longitudinal cut was performed typically angled $15^{\circ}$ from dorsal-medial to plantar-lateral to displace the capital fragment plantarly by 1 to $2 \mathrm{~mm}$. It was essential to avoid plunging beyond the lateral cortex where the deep plantar branch of the dorsalis pedis artery penetrates the first intermetatarsal space. The wires were withdrawn and the dorsal and plantar cuts were completed. Care was taken to avoid the first tarsometatarsal joint by staying parallel to the wire path while making the proximal cut. Before displacement, the completed cuts were ensured to avoid intraoperative fracture. The correction was obtained with rotation and lateral translation of the plantar fragment while maintaining adequate contact between metaphyseal bone surfaces to prevent troughing, which is when the cortices wedge into the softer cancellous bone of the metatarsal shaft (Fig. 2). Temporary fixation was achieved with Kirschner wires. Bone clamps were avoided to again minimize the occurrence of troughing and fracture. The osteotomy was fixed using two to three $2.4-\mathrm{mm}$ or $2.7-\mathrm{mm}$ countersunk lagged screws placed dorsal to plantar avoiding too lateral a position to avert possible postoperative stress fracture. The size and number of screws varied depending on size and length of the metatarsal shaft. The screw heads were placed flush to prevent prominent hardware using controlled compression [20]. Once the screw engaged the plantar cortex, compression was continued until the dorsal cortices were level [20]. The redundant bone on the medial side of the metatarsal head and shaft was resected with the saw and used to bone graft the gaps at the proximal and distal cuts caused by fragment rotation. Concomitant procedures included Akin (four), proximal interphalangeal joint resection (12), neuroma excision (two), and lesser metatarsal shortening (one).

The postoperative protocol allowed immediate weightbearing on the heel or lateral side of the foot in a postoperative shoe with crutches for balance during the first 2 weeks. Once wounds were healed and sutures removed, active and assisted motion of the metatarsophalangeal joint was permitted. The patients were wearing sneakers at 4 to 6 weeks, depending on the amount of swelling.

The AOFAS hallux score questionnaire was completed at the preoperative assessment and at the final followup. This widely used scoring system consists of a 100-point scale divided into three main categories: pain, function, and alignment. The pain and function portions were answered by the patient. The physical examination items were completed by the primary surgeon (MJO). Higher scores indicate better function [3].

The patients received an additional questionnaire at final followup. They were asked if they were satisfied with the

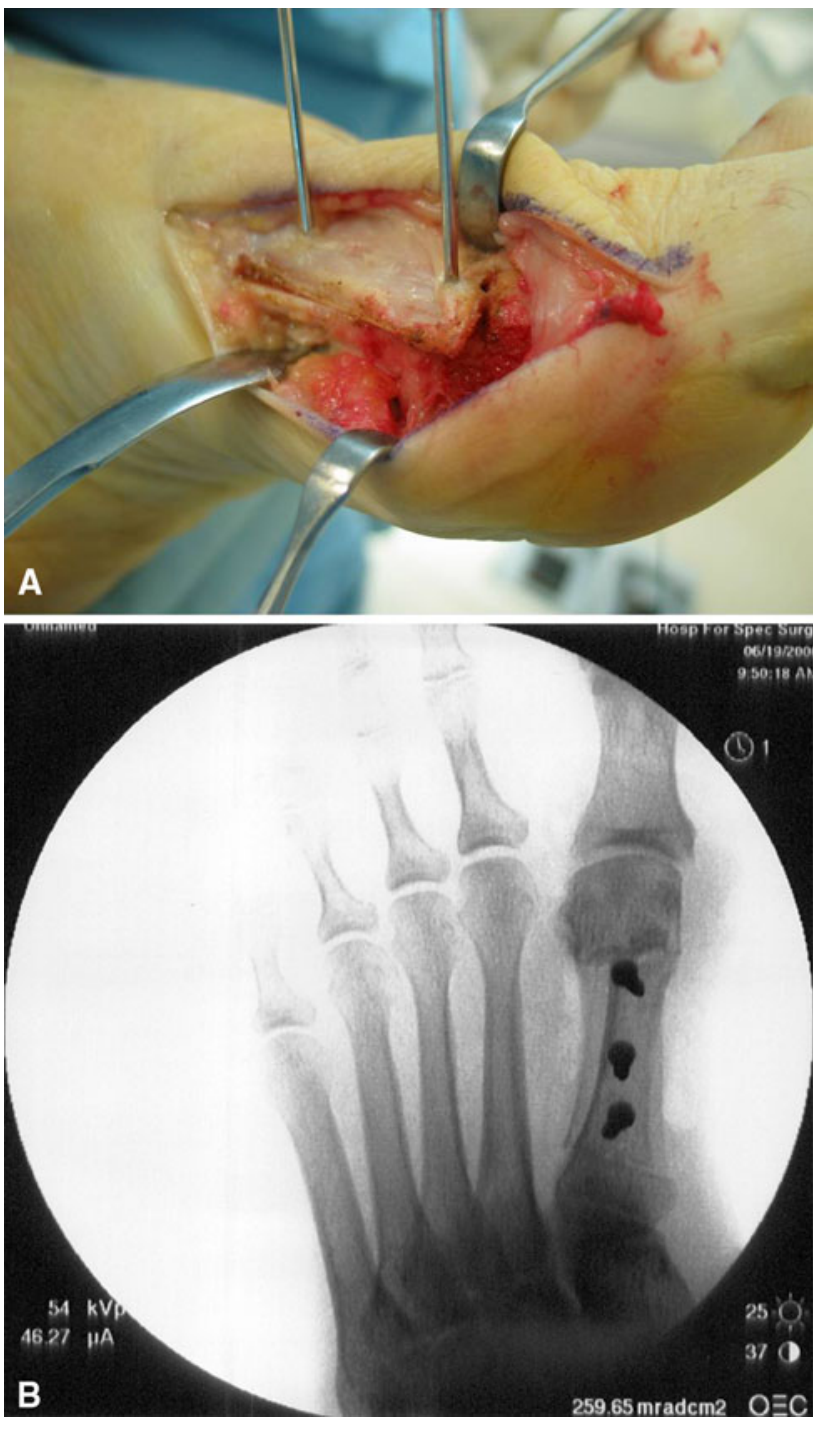

Fig. 2A-B (A) A clinical photograph and (B) a radiograph show the correction obtained by rotation and lateral translation of the plantar fragment while maintaining adequate contact between metaphyseal bone surfaces.

results of the surgery, satisfied with reservations, or unsatisfied, and if they would have the procedure again and recommend the procedure to a friend.

Radiographic assessment was performed using weightbearing dorsoplantar and lateral radiographs preoperatively and postoperatively at 2, 6, and 12 weeks (Fig. 3) and reviewed by one individual (SCC). The HVA and the IMA were measured. The HVA was assessed using the intersection of the bisection of the first metatarsal and proximal phalanx. Normal values for the HVA range from $10^{\circ}$ to $15^{\circ}$ [13]. The IMA was measured as the angle formed by the intersection of the bisection of the first and second metatarsals. Normal values for the IMA range from $7^{\circ}$ to $9^{\circ}$ [13]. 


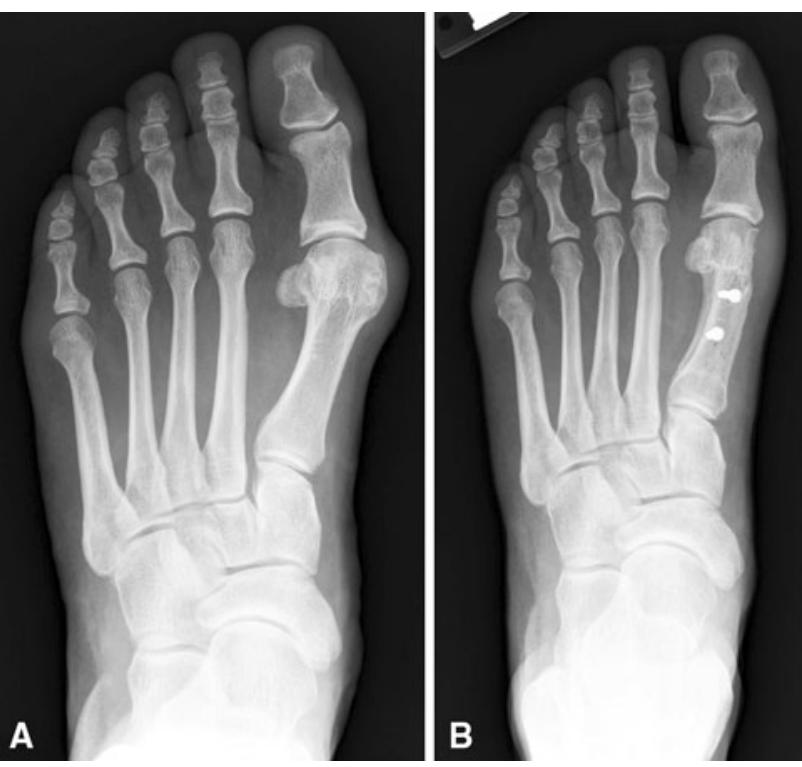

Fig. 3A-B (A) A preoperative AP radiograph shows a moderate metatarsus primus varus and hallux valgus deformity. (B) A postoperative radiograph shows adequate correction of the HVA and IMA.

The preoperative data were compared with the results at followup using the paired Student's t test at the 95\% significance level $(\mathrm{p}<0.05)$.

\section{Results}

The average preoperative AOFAS score of 61.5 (range, 34-76) improved $(\mathrm{p}<0.0001)$ to 90.3 (range, 58-100), indicating an acceptable functional restoration of the forefoot. Patients returned to wearing dress shoes in 3.4 months (Fig. 4). Seventy-one percent of patients were satisfied, 23\% were satisfied with reservations, and $6 \%$ were not satisfied with their results. Eighty-five percent would have the procedure again and $88 \%$ would recommend the procedure to a friend.

The average preoperative HVA of $34.6^{\circ}$ (range, $\left.18^{\circ}-55^{\circ}\right)$ improved $(\mathrm{p}<0.0001)$ to $14.9^{\circ}$ (range, $\left.4^{\circ}-33^{\circ}\right)$, and the average preoperative IMA of $15.8^{\circ}$ (range, $\left.10^{\circ}-24^{\circ}\right)$ improved $(\mathrm{p}<0.0001)$ to $7.2^{\circ}\left(\right.$ range, $\left.0^{\circ}-15^{\circ}\right)$. Two feet had recurrence of hallux valgus deformity.

Complications included recurrence in two patients. These both occurred in our early experience of performing the osteotomy with more translation than rotation of the plantar-distal fragment. We now use more rotation combined with translation to obtain adequate correction. Troughing was noted in three patients and resulted in stiffness of the first metatarsophalangeal joint. These patients were older and tolerated the decreased ROM. No repeat procedures were necessary. One patient had a wound infection develop that resolved with oral antibiotics.
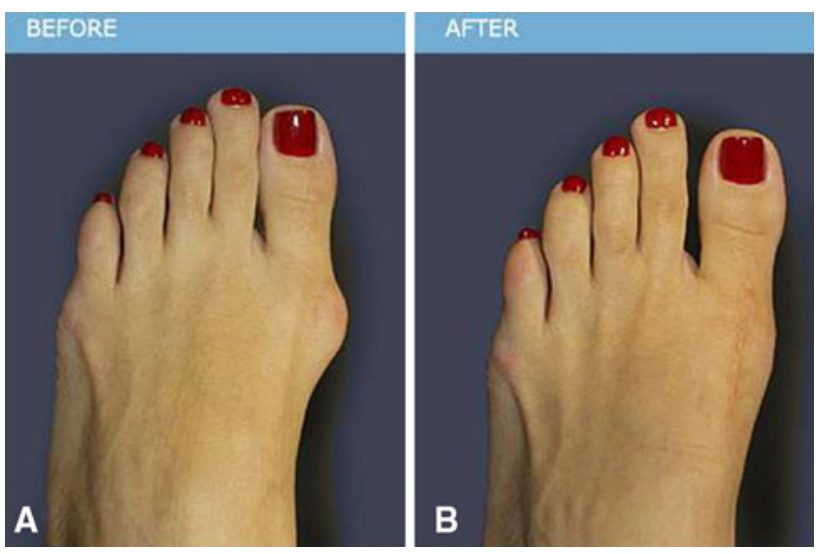

Fig. 4A-B Photographs (A) before and (B) after surgery show the improvement in the appearance of the foot.

\section{Discussion}

The scarf osteotomy to correct hallux valgus deformity can provide a predictable and satisfying outcome for the patient and the surgeon $[14,23]$ with a low rate of complications $[1,11,13]$. In our study, we asked the following questions: (1) Does the scarf osteotomy result in improved AOFAS scores and patient satisfaction? (2) Will the scarf osteotomy alone correct radiographic parameters, ie, HVA and IMA? (3) What are the associated complications of this procedure? (4) What are some technical pearls in surgical technique to obtain good correction and avoid such complications?

One of the limitations of this study was that we had incomplete preoperative AOFAS scores and/or radiographs for 17 patients resulting in their exclusion from the study. The other critique of this study could be the average followup of 26.4 months providing no information regarding long-term consequences of the procedure.

We found the preoperative AOFAS score improved postoperatively by 40 points, with $85 \%$ of patients stating they would have the procedure again and $94 \%$ of patients satisfied. Other studies have showed similar improvements in AOFAS scores: Kristen et al. 50.1 to 91 [14], Lipscombe et al. 47.9 to 96.1 [15], and Jones et al. 52 to 89 [13].

This Z-step cut provides versatility and reliability enabling one to address a wide range of hallux valgus deformities as seen in our patient population. Our results show excellent correction of HVA and IMA, also comparable to rates reported in the literature $[13,15]$.

A proximal phalanx osteotomy (Akin) has been reported to have been combined with the scarf osteotomy and distal soft tissue realignment in $70 \%$ to $100 \%$ of cases $[7,13,16]$. Unlike other studies, a proximal phalanx osteotomy (Akin) was added to only four of our cases to provide additional correction. We attribute this finding to our scarf technique using rotation in combination with translation of the 
Fig. 5A-B (A) Troughing is when the cortices wedge into the softer cancellous bone of the metatarsal shaft $(\mathbf{B})$ resulting in elevation of the first ray.
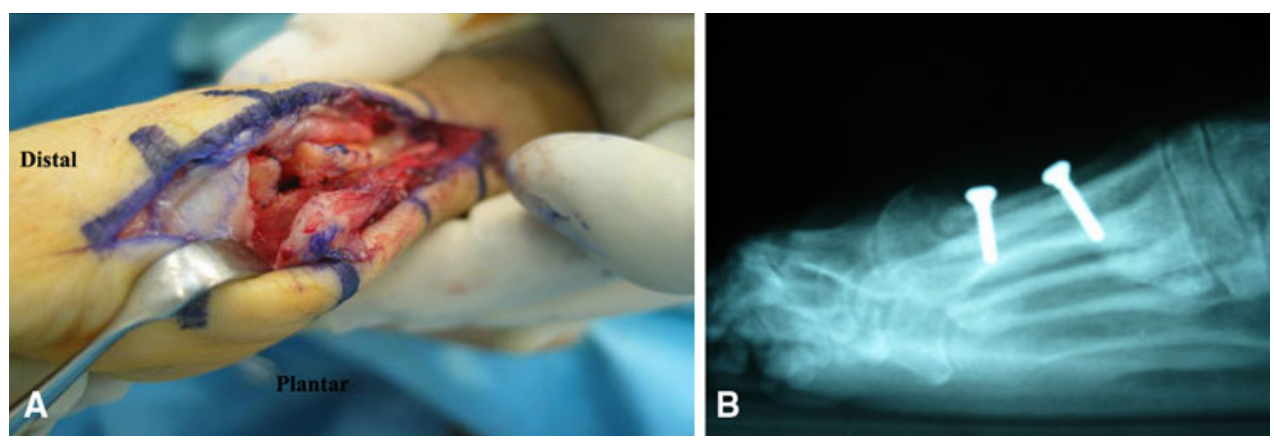

plantar-distal metatarsal fragment obtaining good correction without the need for an Akin osteotomy.

The scarf osteotomy is our preferred osteotomy for hallux valgus deformity, but it is not without complications. Complications, intraoperative and postoperative, have been reported at variable incidences, including troughing of the metatarsal, fracture, delayed union, rotational malunion, recurrence of deformity, and infection $[4,12,14,20,21]$. The nature of the osteotomy is more technically challenging, has a higher surgical learning curve, and requires meticulous attention to the threedimensional changes [17]. Smith et al. [21] reported a $6 \%$ perioperative complication rate from their early experience to create awareness for those beginning to master the scarf osteotomy. Before making modifications in his osteotomy, Coetzee [4] reported a complication rate of $47 \%$. This includes troughing in $35 \%$ [4].

Troughing is one of the most common complications of the scarf osteotomy [4], and it occurs when the cortices wedge into the softer cancellous bone of the metatarsal shaft resulting in elevation of the first ray (Fig. 5). This, in turn, can lead to stiffness of the first metatarsophalangeal joint with subsequent arthrosis or possible lateral metatarsalgia and stress fracture. Troughing can be prevented by avoiding purely diaphyseal cuts, limiting the distal and proximal step-cut to 2 to $3 \mathrm{~mm}$ in length, and keeping the distal cut at the junction of the dorsal and middle third of the metatarsal shaft. Most importantly, the plantar fragment of the metatarsal shaft should be rotated more than translated in relation to the dorsal fragment. This technique will maintain better cortical apposition, thus preventing the cortices from wedging into the cancellous bone. For patients with osteoporosis, use of the medial eminence resection as bone graft for the cancellous bone in the medullary canal is often absent.

A followup study by Coetzee et al. [5] describes the changes made to Coetzee's operative technique, including limiting the short arms of the $\mathrm{Z}$ osteotomy to 2 to $3 \mathrm{~mm}$ in depth, avoiding patients with osteoporosis, and adjusting the direction of the long arm of the $\mathrm{Z}$ osteotomy. After these modifications, their overall complication rate decreased with a $1 \%$ incidence of troughing.

The scarf osteotomy is an effective technique for hallux valgus deformity providing tremendous inherent stability, thus retaining the benefit of early mobilization [11]. This osteotomy also provides precise control over metatarsal length, elevation, and position but is technically demanding. Our surgical technique incorporates technical pearls from the literature $[2,6,20]$, and our own, to minimize pitfalls.

\section{References}

1. Aminian A, Kelikian A, Moen T. Scarf osteotomy for hallux valgus deformity: an intermediate followup of clinical and radiographic outcomes. Foot Ankle Int. 2006;27:883-886.

2. Barouk LS. Scarf osteotomy for hallux valgus correction: local anatomy, surgical technique, and combination with other forefoot procedures. Foot Ankle Clin. 2000;5:525-558.

3. Baumhauer JF, Nawoczenski DA, DiGiovanni BF, Wilding GE. Reliability and validity of the American Orthopaedic Foot and Ankle Society Clinical Rating Scale: a pilot study for the hallux and lesser toes. Foot Ankle Int. 2006;27:1014-1019.

4. Coetzee JC. Scarf osteotomy for hallux valgus repair: the dark side. Foot Ankle Int. 2003;24:29-33.

5. Coetzee JC, Pomeroy GC, Watts JD, Barrow C. The use of autologous concentrated growth factors to promote syndesmosis fusion in the Agility total ankle replacement: a preliminary study. Foot Ankle Int. 2005;26:840-846.

6. Coetzee JC, Rippstein P. Surgical strategies: scarf osteotomy for hallux valgus. Foot Ankle Int. 2007;28:529-535.

7. Crevoisier X, Mouhsine E, Ortolano V, Udin B, Dutoit M. The scarf osteotomy for the treatment of hallux valgus deformity: a review of 84 cases. Foot Ankle Int. 2001;22:970-976.

8. Dereymaeker G. Scarf osteotomy for correction of hallux valgus: surgical technique and results as compared to distal chevron osteotomy. Foot Ankle Clin. 2000;5:513-524.

9. Dhukaram V, Hullin MG, Senthil Kumar C. The Mitchell and Scarf osteotomies for hallux valgus correction: a retrospective, comparative analysis using plantar pressures. J Foot Ankle Surg. 2006;45:400-409.

10. Gallentine JW, Deorio JK, Deorio MJ. Bunion surgery using locking-plate fixation of proximal metatarsal chevron osteotomies. Foot Ankle Int. 2007;28:361-368.

11. Gupta S, Fazal MA, Williams L. Minifragment screw fixation of the Scarf osteotomy. Foot Ankle Int. 2008;29:385-389. 
12. Jones AL. Recombinant human bone morphogenic protein-2 in fracture care. J Orthop Trauma. 2005;19(10 suppl):S23-S25.

13. Jones S, Al Hussainy HA, Ali F, Betts RP, Flowers MJ. Scarf osteotomy for hallux valgus: a prospective clinical and pedobarographic study. J Bone Joint Surg Br. 2004;86:830-836.

14. Kristen KH, Berger C, Stelzig S, Thalhammer E, Posch M, Engel A. The SCARF osteotomy for the correction of hallux valgus deformities. Foot Ankle Int. 2002;23:221-229.

15. Lipscombe S, Molloy A, Sirikonda S, Hennessy MS. Scarf osteotomy for the correction of hallux valgus: midterm clinical outcome. J Foot Ankle Surg. 2008;47:273-277.

16. Lorei TJ, Kinast C, Klarner H, Rosenbaum D. Pedographic, clinical, and functional outcome after scarf osteotomy. Clin Orthop Relat Res. 2006;451:161-166.

17. Nyska M, Trnka HJ, Parks BG, Myerson MS. Proximal metatarsal osteotomies: a comparative geometric analysis conducted on sawbone models. Foot Ankle Int. 2002;23:938-945.
18. Okuda R, Kinoshita M, Morikawa J, Jotoku T, Abe M. Distal soft tissue procedure and proximal metatarsal osteotomy in hallux valgus. Clin Orthop Relat Res. 2000;379:209-217.

19. Sammarco VJ, Acevedo J. Stability and fixation techniques in first metatarsal osteotomies. Foot Ankle Clin. 2001;6:409-432, v-vi.

20. Saragas NP. Technique tip: preventing "troughing" with the scarf osteotomy. Foot Ankle Int. 2005;26:779-780.

21. Smith AM, Alwan T, Davies MS. Perioperative complications of the scarf osteotomy. Foot Ankle Int. 2003;24:222-227.

22. Trnka HJ, Parks BG, Ivanic G, Chu IT, Easley ME, Schon LC, Myerson MS. Six first metatarsal shaft osteotomies: mechanical and immobilization comparisons. Clin Orthop Relat Res. 2000; 381:256-265.

23. Weil LS. Scarf osteotomy for correction of hallux valgus: historical perspective, surgical technique, and results. Foot Ankle Clin. 2000;5:559-580. 